\title{
ON A QUESTION OF RADEMACHER CONCERNING DEDEKIND SUMS
}

\author{
LAURENCE PINZUR
}

\begin{abstract}
Rademacher raised the following question concerning the ordinary Dedekind sum $s(h, k)$. If $h_{1} / k_{1}$ and $h_{2} / k_{2}$ are adjacent Farey fractions such that $s\left(h_{1}, k_{1}\right)>0$ and $s\left(h_{2}, k_{2}\right)>0$, is it necessarily true that $s\left(h_{1}+h_{2}, k_{1}+k_{2}\right) \geqslant 0$ ? The answer to this question is found to be no. In fact, a characterization of all pairs of adjacent Farey fractions where the answer to Rademacher's question is no is given.
\end{abstract}

The ordinary Dedekind sum $s(h, k)$ is defined for relatively prime positive integers $h$ and $k$ by

$$
s(h, k)=\sum_{n=1}^{k-1} \frac{n}{k}\left(\left(\frac{n h}{k}\right)\right)
$$

where

$$
((x))= \begin{cases}x-[x]-\frac{1}{2}, & \text { if } x \notin \mathbf{Z}, \\ 0, & \text { if } x \in \mathbf{Z} .\end{cases}
$$

Rademacher raised the following question at the number theory conference held in 1963 at the University of Colorado [3, p. 112]. Let $h_{1} / k_{1}$ and $h_{2} / k_{2}$ be adjacent Farey fractions with $s\left(h_{1}, k_{1}\right)>0$ and $s\left(h_{2}, k_{2}\right)>0$. Is it always true that $s\left(h_{1}+h_{2}, k_{1}+k_{2}\right) \geqslant 0$ ? The answer to this question is no, and an infinite class of examples is given by the following pairs of Farey fractions:

$$
\frac{h_{1}}{k_{1}}=\frac{3}{3 m+1}, \quad \frac{h_{2}}{k_{2}}=\frac{3 m-4}{3 m^{2}-3 m-1} \quad(m \in \mathbf{Z}, m \geqslant 4) .
$$

In an earlier version of this paper, the proof was by use of the following explicit formulae:

$$
\begin{gathered}
s(3,3 m+1)=3 m(m-3) / 12(3 m+1), \\
s\left(3 m-4,3 m^{2}-3 m-1\right)=(m+1)(m-2) / 4\left(3 m^{2}-s m-1\right),
\end{gathered}
$$

and

$$
s\left(3 m-1,3 m^{2}\right)=-1 / 18 m^{2} .
$$

These formulae follow from repeated use of the well-known reciprocity theorem for Dedekind sums [4, p. 4], and the fact that $h \equiv h^{\prime}(\bmod k)$ implies that $s(h, k)=s\left(h^{\prime}, k\right)$. In this paper we not only deduce this result from a

Presented to the Society, December 3, 1975; received by the editors December 31, 1975 and, in revised form, March 3, 1976.

AMS (MOS) subject classifications (1970). Primary 10A40. 
recent result of Hickerson (see Theorem A below), but we also characterize all pairs of adjacent Farey fractions for which the answer to Rademacher's question is no.

Throughout this paper, we will denote by $\left[a_{1}, \ldots, a_{n}\right]$ the simple continued fraction $\left[0 ; a_{1}, \ldots, a_{n}\right]$ with partial quotients $a_{1}, \ldots, a_{n}$. For a fixed continued fraction $\left[a_{1}, \ldots, a_{n}\right]$, let

$$
A=\sum_{i=1 ; i \text { odd }}^{n} a_{i}-\sum_{i=1 ; i \text { even }}^{n} a_{i}
$$

The following two sets of conditions will play an important role in the characterization theorem:

$$
\begin{aligned}
& \left\{\begin{array}{l}
A=0, \\
{\left[a_{1}, \ldots, a_{n}\right]>\left[a_{n}, \ldots, a_{1}\right],} \\
a_{n}>3-\left[a_{1}, \ldots, a_{n-1}\right]-\left[a_{n-1}, \ldots, a_{1}\right] ;
\end{array}\right. \\
& \left\{\begin{array}{l}
A=1, \\
{\left[a_{1}, \ldots, a_{n}+1\right]<\left[a_{n}+1, \ldots, a_{1}\right],} \\
a_{n}>2-\left[a_{1}, \ldots, a_{n-1}\right]-\left[a_{n-1}, \ldots, a_{1}\right] .
\end{array}\right.
\end{aligned}
$$

We can now state the main result.

THEOREM. Let $h_{1}<k_{1}$ and $h_{2}<k_{2}$ be positive integers with $2 \leqslant k_{1}<k_{2}$. Then $h_{1} / k_{1}$ and $h_{2} / k_{2}$ are adjacent Farey fractions of order $k_{2}$ with $s\left(h_{1}, k_{1}\right)>$ $0, s\left(h_{2}, k_{2}\right)>0$, and $s\left(h_{1}+h_{2}, k_{1}+k_{2}\right)<0$ if and only if there exist positive integers $a_{1}, \ldots, a_{n}(n \geqslant 4$, even) satisfying either (2) or (3) and both of the following conditions:

(i) $h_{1} / k_{1}=\left[a_{1}, \ldots, a_{n-1}\right]$,

(ii) $h_{2} / k_{2}=\left[a_{1}, \ldots, a_{n}\right]$.

The proof relies heavily upon the following recent result of Hickerson [1].

THEOREM A. If $\left[\alpha_{0} ; \alpha_{1}, \ldots, \alpha_{r}\right]$ is the simple continued fraction expansion of $h / k$, then

$$
\begin{aligned}
s(h, k)= & \frac{-1+(-1)^{r}}{8} \\
& +\frac{1}{12}\left(\left[0 ; \alpha_{1}, \ldots, \alpha_{r}\right]+(-1)^{r+1}\left[0 ; \alpha_{r}, \ldots, \alpha_{1}\right]\right. \\
& \left.\quad+\alpha_{1}-\alpha_{2}+\cdots+(-1)^{r+1} \alpha_{r}\right) .
\end{aligned}
$$

We also need two simple facts which we state as lemmas.

It is well known [2, pp. 154-155] that if $a_{1}, \ldots, a_{n}$ are positive integers $(n \geqslant 2)$ and if $h_{1} / k_{1}$ and $h_{2} / k_{2}$ are the reduced rational numbers whose simple continued fraction expansions are $\left[a_{1}, \ldots, a_{n-1}\right]$ and $\left[a_{1}, \ldots, a_{n}\right]$, respectively, then $h_{1} / k_{1}$ and $h_{2} / k_{2}$ are adjacent Farey fractions of order $k_{2}$. In some sense the converse is also true.

LEMMA 1. If $h_{1} / k_{1}$ and $h_{2} / k_{2}$ are adjacent Farey fractions of order $k_{2}$, then there exist positive integers $a_{1}, \ldots, a_{n}$ such that $h_{1} / k_{1}=\left[a_{1}, \ldots, a_{n-1}\right]$ and $h_{2} / k_{2}=\left[a_{1}, \ldots, a_{n}\right]$. 
Proof. If $h_{1} / k_{1}<h_{2} / k_{2}$, let $\left[a_{1}, \ldots, a_{n}\right]$ be the unique simple continued fraction expansion of $h_{2} / k_{2}$ with $n$ odd. Note that $n \neq 1$, since $k_{1}<k_{2}$. Then $h_{1} / k_{1}=\left[a_{1}, \ldots, a_{n-1}\right]$ is the adjacent Farey fraction of order $k_{2}$ not exceeding $h_{2} / k_{2}$. If $h_{1} / k_{1}>h_{2} / k_{2}$, let $\left[a_{1}, \ldots, a_{n}\right]$ be the unique simple continued fraction expansion of $h_{2} / k_{2}$ with $n$ even. Then $h_{1} / k_{1}=\left[a_{1}, \ldots, a_{n-1}\right]$ has the desired properties.

Lemma 2. If $p_{i} / q_{i}=\left[a_{1}, \ldots, a_{i}\right]$ for $i=1,2, \ldots, n$, then

$$
\frac{p_{n-1}+p_{n}}{q_{n-1}+q_{n}}=\left[a_{1}, \ldots, a_{n}, 1\right]=\left[a_{1}, \ldots, a_{n}+1\right] \text {. }
$$

This is a special case of a theorem in [2, p. 154].

Proof of TheOREM. First suppose that there exist integers $a_{1}, \ldots, a_{n}$ which satisfy the conditions stated in the theorem. Then by (i) and (ii), we know that $h_{1} / k_{1}$ and $h_{2} / k_{2}$ are adjacent Farey fractions of order $k_{2}$. Now using Theorem A and Lemma 2, we obtain

$$
\begin{gathered}
12 \cdot s\left(h_{1}, k_{1}\right)=-3+\left[a_{1}, \ldots, a_{n-1}\right]+\left[a_{n-1}, \ldots, a_{1}\right]+A+a_{n} \\
12 \cdot s\left(h_{2}, k_{2}\right)=\left[a_{1}, \ldots, a_{n}\right]-\left[a_{n}, \ldots, a_{1}\right]+A
\end{gathered}
$$

and

$$
\begin{aligned}
12 \cdot s\left(h_{1}+h_{2}, k_{1}+k_{2}\right)= & {\left[a_{1}, \ldots, a_{n}+1\right] } \\
& -\left[a_{n}+1, \ldots, a_{1}\right]+A-1,
\end{aligned}
$$

where $A$ is as defined in (1). If (2) holds, then (4), (5) and (6) show that

$$
\begin{gathered}
12 \cdot s\left(h_{1}, k_{1}\right)=-3+\left[a_{1}, \ldots, a_{n-1}\right]+\left[a_{n-1}, \ldots, a_{1}\right]+a_{n}>0, \\
12 \cdot s\left(h_{2}, k_{2}\right)=\left[a_{1}, \ldots, a_{n}\right]-\left[a_{n}, \ldots, a_{1}\right]>0,
\end{gathered}
$$

and

$$
12 \cdot s\left(h_{1}+h_{2}, k_{1}+k_{2}\right)=\left[a_{1}, \ldots, a_{n}+1\right]-\left[a_{n}+1, \ldots, a_{1}\right]-1<0,
$$

respectively. The last inequality follows from the fact that the bracketed quantities are in $(0,1)$. If (3) holds, then by a similar argument we find that

$$
\begin{gathered}
12 \cdot s\left(h_{1}, k_{1}\right)=-2+\left[a_{1}, \ldots, a_{n-1}\right]+\left[a_{n-1}, \ldots, a_{1}\right]+a_{n}>0, \\
12 \cdot s\left(h_{2}, k_{2}\right)=\left[a_{1}, \ldots, a_{n}\right]-\left[a_{n}, \ldots, a_{1}\right]+1>0,
\end{gathered}
$$

and

$$
12 \cdot s\left(h_{1}+h_{2}, k_{1}+k_{2}\right)=\left[a_{1}, \ldots, a_{n}+1\right]-\left[a_{n}+1, \ldots, a_{1}\right]<0 .
$$

Hence the given conditions on $a_{1}, a_{2}, \ldots, a_{n}$ are sufficient to ensure the existence of positive integers $h_{1}, k_{1}, h_{2}$, and $k_{2}$ with the desired properties.

Conversely, suppose that $h_{1} / k_{1}$ and $h_{2} / k_{2}$ are adjacent Farey fractions with $2 \leqslant k_{1}<k_{2}$ and with the property that $s\left(h_{1}, k_{1}\right)>0, s\left(h_{2}, k_{2}\right)>0$, and $s\left(h_{1}+h_{2}, k_{1}+k_{2}\right)<0$. Let $a_{1}, \ldots, a_{n}$ be those positive integers as described in Lemma 1; i.e. $h_{1} / k_{1}=\left[a_{1}, \ldots, a_{n-1}\right]$ and $h_{2} / k_{2}=\left[a_{1}, \ldots, a_{n}\right]$. We first show that $n$ must be even. If $n$ were odd, we could use Theorem A and Lemma 2 to write 
and

$$
\begin{aligned}
12 \cdot s\left(h_{2}, k_{2}\right)= & -3+\left[a_{1}, \ldots, a_{n}\right]+\left[a_{n}, \ldots, a_{1}\right] \\
& +a_{1}-a_{2}+\cdots+a_{n}>0,
\end{aligned}
$$

$$
\begin{aligned}
12 \cdot s\left(h_{1}+h_{2}, k_{1}+k_{2}\right)= & -3+\left[a_{1}, \ldots, a_{n}+1\right]+\left[a_{n}+1, \ldots, a_{1}\right] \\
& +a_{1}-a_{2}+\cdots+\left(a_{n}+1\right)<0 .
\end{aligned}
$$

This implies that

$$
\left[a_{1}, \ldots, a_{n}+1\right]-\left[a_{1}, \ldots, a_{n}\right]+1<\left[a_{n}, \ldots, a_{1}\right]-\left[a_{n}+1, \ldots, a_{1}\right] \text {. }
$$

The above inequality, however, cannot be true since

$$
\left[a_{1}, \ldots, a_{n}+1\right]-\left[a_{1}, \ldots, a_{n}\right]=\frac{-1}{k_{2}\left(k_{1}+k_{2}\right)} \geqslant \frac{-1}{3(2+3)}=-\frac{1}{15},
$$

and

$$
\left[a_{n}, \ldots, a_{1}\right]-\left[a_{n}+1, \ldots, a_{1}\right] \leqslant\left[a_{n}\right]-\left[a_{n}+2\right]=\frac{1}{a_{n}}-\frac{1}{a_{n}+2} \leqslant \frac{2}{3} .
$$

Hence $n$ must be even, and equations (4), (5), and (6) hold. Since $s\left(h_{2}, k_{2}\right)>$ 0 and $s\left(h_{1}+h_{2}, k_{1}+k_{2}\right)<0$, we can use (5) and (6) above to obtain

$$
\begin{aligned}
{\left[a_{n}, \ldots, a_{1}\right]-\left[a_{1}, \ldots, a_{n}\right] } & \\
<A & <1+\left[a_{n}+1, \ldots, a_{1}\right]-\left[a_{1}, \ldots, a_{n}+1\right] .
\end{aligned}
$$

From this last expression we see that $A$ equals 0 or 1 , since $A$ is an integer, and each of the bracketed quantities are fractions in $(0,1)$. If $A=0$, then using (4) we have $a_{n}>3-\left[a_{1}, \ldots, a_{n-1}\right]-\left[a_{n-1}, \ldots, a_{1}\right]$, since $s\left(h_{1}, k_{1}\right)>$ 0 , and using (5) we have $\left[a_{1}, \ldots, a_{n}\right]>\left[a_{n}, \ldots, a_{1}\right]$, since $s\left(h_{2}, k_{2}\right)>0$. If $A=1$, then using (4) we have $a_{n}>2-\left[a_{1}, \ldots, a_{n-1}\right]-\left[a_{n-1}, \ldots, a_{1}\right]$, and using (6) we have

$$
\left[a_{1}, \ldots, a_{n}+1\right]<\left[a_{n}+1, \ldots, a_{1}\right],
$$

since $s\left(h_{1}+h_{2}, k_{1}+k_{2}\right)<0$.

Finally we show that $n$ cannot equal 2. First suppose that $n=2$ and $A=0$. Then $h_{2} / k_{2}$ has a simple continued fraction expansion of the form $[\alpha, \alpha]$ which implies that $s\left(h_{2}, k_{2}\right)=0$ by Theorem A. Next suppose that $n=2$ and $A=1$. Then $h_{2} / k_{2}$ has a simple continued fraction expansion of the form $[\alpha+1, \alpha]$, and hence by Lemma $2,\left(h_{1}+h_{2}\right) /\left(k_{1}+k_{2}\right)$ has a simple continued fraction expansion of the form $[\alpha+1, \alpha+1]$. Once again by Theorem A we then have $s\left(h_{1}+h_{2}, k_{1}+k_{2}\right)=0$. This contradiction shows that $n \neq 2$ and the proof is complete.

Consider, for example, the simple continued fraction expansions of the pairs of Farey fractions given in the first paragraph of the paper:

$$
h_{1} / k_{1}=3 /(3 m+1)=[m, 2,1]
$$

and

$$
h_{2} / k_{2}=(3 m-4) /\left(3 m^{2}-3 m-1\right)=[m, 2,1, m-2] .
$$

It is easily verified that for $m \geqslant 4,\left(a_{1}, a_{2}, a_{3}, a_{4}\right)=(m, 2,1, m-2)$ satisfy all the requirements of the theorem. Another interesting example is found by 
considering certain convergents of the simple continued fraction expansion of the number $(315-\sqrt{ } 5) / 410$. This quadratic irrational has the periodic continued fraction expansion $[1,3,4, \overline{1}]$. Denote the $i$ th convergent to this number by $p_{i} / q_{i}$. If we now set $h_{j}=p_{2 j+3}$ and $k_{j}=q_{2 j+3}$ for all positive integers $j$, then we have

$$
\begin{aligned}
& h_{1} / k_{1}=[1,3,4,1,1]=[1,3,4,2]=29 / 38, \\
& h_{2} / k_{2}=[1,3,4,1,1,1,1]=[1,3,4,1,1,2]=74 / 97, \\
& h_{3} / k_{3}=[1,3,4,1,1,1,1,1,1]=[1,3,4,1,1,1,1,2]=193 / 253,
\end{aligned}
$$

and so on. We now apply the theorem repeatedly with $\left(a_{1}, a_{2}, \ldots, a_{6}\right)=$ $(1,3,4,1,1,2)$ first, then $\left(a_{1}, a_{2}, \ldots, a_{8}\right)=(1,3,4,1,1,1,1,2)$, and so on. Observe that if we drop the last partial quotient of $h_{j+1} / k_{j+1}$ we obtain $h_{j} / k_{j}$. Hence we have an infinite sequence, $\left\{h_{j} / k_{j}\right\}_{j=1}^{\infty}$, of rational numbers such that for all positive integers $j, h_{j} / k_{j}$ and $h_{j+1} / k_{j+1}$ are adjacent Farey fractions of order $k_{j+1}$,

$$
s\left(h_{j}, k_{j}\right)>0, \quad s\left(h_{j+1}, k_{j+1}\right)>0 \text { and } s\left(h_{j}+h_{j+1}, k_{j}+k_{j+1}\right)<0 .
$$

NOTE ADDED IN PROOF. At the January 1976 AMS meeting at San Antonio, Kenneth Rosen presented a paper (731-10-24) which exhibited infinitely many pairs of adjacent Farey fractions for which the answer to Rademacher's question is no. That result is contained in the present paper, and, in fact, Rosen's class of examples is obtained when $\left(a_{1}, a_{2}, a_{3}, a_{4}\right)=(m-1,1,2, m)$ for $m \in \mathbf{Z}, m \geqslant 3$.

\section{REFERENCES}

1. D. Hickerson, Continued fractions and density results for Dedekind sums (preprint).

2. I. Niven and H. Zuckerman, An introduction to the theory of numbers, 3rd ed., Wiley, New York, 1972. MR 49 \#8921.

3. H. Rademacher, Proc. 1963 Number Theory Conf., Univ. of Colorado, Boulder, Colo., 1963.

4. H. Rademacher and E. Grosswald, Dedekind sums, Carus Math. Monos., no. 16, Math. Assoc. Amer., Washington, D. C., 1972. 